\section{Pathogenese, Diagnostik und Therapie der COPD}

\section{Epidemiologie}

Die Prävalenz der COPD (=chronic obstructive pulmonary disease) liegt um 5\%. In Europa ist sie die dritthäufigste, in den USA die vierthäufigste Todesursache $[3,56]$. Der starke Anstieg der Mortalitäts-, vor allem aber der Morbiditätsraten in den letzten 30 Jahren spiegelt Trends der Tabakepidemie wider. Die starken Raucher der Kriegs- und Nachkriegsjahre haben jetzt ein höheres Lebensalter mit manifesten Spätschäden des Zigarettenrauchens erreicht. Der in den Nachkriegsjahren stetig steigende Anteil rauchender Frauen bedingt aktuell eine im Vergleich zu den Männern stärkere Zunahme der COPD-Prävalenz. Zwar ist der Zigarettenkonsum von erwachsenen Frauen und Männern in den Industrienationen stagnierend bis rückläufig, aufgrund der langen Latenz bis zum Auftreten klinischer Symptome wird sich dies aber erst nach Jahren auf die COPD-Inzidenz auswirken. Im Gegensatz zu den erwachsenen Rauchern ermitteln aktuelle Umfragen in der Schweiz bei den Jugendlichen einen kontinuierlichen Anstieg des Nikotinkonsums. Bei den 15- bis 16jährigen überwiegen die Mädchen sogar. Diese Daten betonen die Bedeutung der Tabakprävention im Hinblick auf eine Senkung der COPD-Häufigkeit [65]. Die Belastung des Gesundheitswesens ist beträchtlich [10]. COPD ist in den USA die zweithäufigste Ursache für Rentenzahlungen an Invalide mit chronischen Erkrankungen, in Deutschland belaufen sich die jährlichen direkten und indirekten Krankheitskosten auf über $13 \mathrm{Mrd}$. DM.

\section{Definitionen}

Charakteristikum der COPD ist eine chronische exspiratorische Atemflußbehinderung, die sich lungenfunktionell durch einen persistierend unter 70\% verminderten Tiffeneau-Quotienten manifestiert. Der Schweregrad wird dagegen am $\mathrm{FEV}_{1}$ (\% Soll) beurteilt (Tab. 1). Grundkrankheiten, die das Syndrom COPD verursachen können, sind die chronische Bronchitis und das Lungenemphysem. Seltener sind Kombinationen mit Aspekten des Asthma bronchiale (z.B. Reversibilität, bronchiale Hyperreaktivität). Trotz klinischer und lungenfunktioneller Überlappungen kann ein Asthma bronchiale aber aufgrund pathophysiologischer und allergologischer Unterschiede meist abgegrenzt werden [27,66]. Ein nichtproportionales Venn-Diagramm skizziert dieses heterogene Patienten-

Pneumologie 2000; 54: 123-132

(C) Georg Thieme Verlag Stuttgart · New York ISSN 0934-8387

\section{E. Ullmer ${ }^{1}$, M. Solèr ${ }^{2}$, H. Hamm¹, A. P. Perruchoud ${ }^{2}$}

${ }^{1}$ Abteilung für Pneumologie, Robert-Koch-Klinik, Medizinische Universitätsklinik Freiburg i. Br., Freiburg, Deutschland

2 Abteilung für Pneumologie, Departement Innere Medizin A, Kantonsspital Basel, Basel, Schweiz

kollektiv, die Subpopulation mit einer COPD ist schraffiert dargestellt (Abb. 1, [3]).

Die chronische Bronchitis ist klinisch definiert als chronisch vermehrte Schleimproduktion mit produktivem Husten über mindestens 3 Monate Dauer pro Jahr in mindestens 2 aufeinanderfolgenden Jahren. Mechanismus der Obstruktion ist eine Hypertrophie und Überfunktion des schleimbildenden Apparates bei gleichzeitig gestörter mukoziliärer Clearance, verbunden mit einer neutrophilen Schleimhautentzündung. Die nachfolgende, als „airway remodelling“ bezeichnete peribronchiale Fibrose mit Verengung der kleinen Luftwege führt zu einer fixierten Atemflußbehinderung.

Das Lungenemphysem ist anatomisch definiert als irreversible Erweiterung der Atemwege distal der Bronchioli terminales mit Zerstörung der Alveolarsepten ohne relevante Fibrosierung. Morphologisch werden drei Formen unterschieden:

Beim panazinären Emphysem ist die Alveolardestruktion gleichmäßig über die Azini verteilt und hat keinen Bezug zu den kleinen Atemwegen. Diese Form der Parenchymschädigung ist vorwiegend in den basalen Lungenabschnitten lokalisiert und tritt z.B. im Rahmen eines $\alpha_{1}$-AntitrypsinMangels auf (Abb. 2a).

Tab. 1 Schweregrade der COPD. Eine obstruktive Ventilationsstörung ist definiert als Verminderung des Tiffeneau-Quotienten ( $\mathrm{FEV}_{1} / \mathrm{FVC}$ ) unter $70 \%$. Der Schweregrad der Obstruktion wird am FEV 1 (\%Soll) beurteilt

FEV $_{1}$ (\%Soll) häufige, jedoch nicht obligate Begleitsymptome oder Befunde

\begin{tabular}{lll}
\hline leicht $>70$ & unproduktiver Husten \\
mittelschwer 51-69 & produktiver Husten \\
& Belastungsdyspnoe + bis ++ \\
& Hypoxämie \\
& leicht erniedrigte Diffusionskapazität* \\
& Belastungsdyspnoe ++ bis +++ \\
schwer & Bronchospasmus \\
& Zyanose \\
& Lungenüberblähung \\
& stark erniedrigte Diffusionskapazität* \\
& Polyglobulie \\
& Hyperkapnie \\
& Rechtsherzinsuffizienz
\end{tabular}

* beim Emphysem 


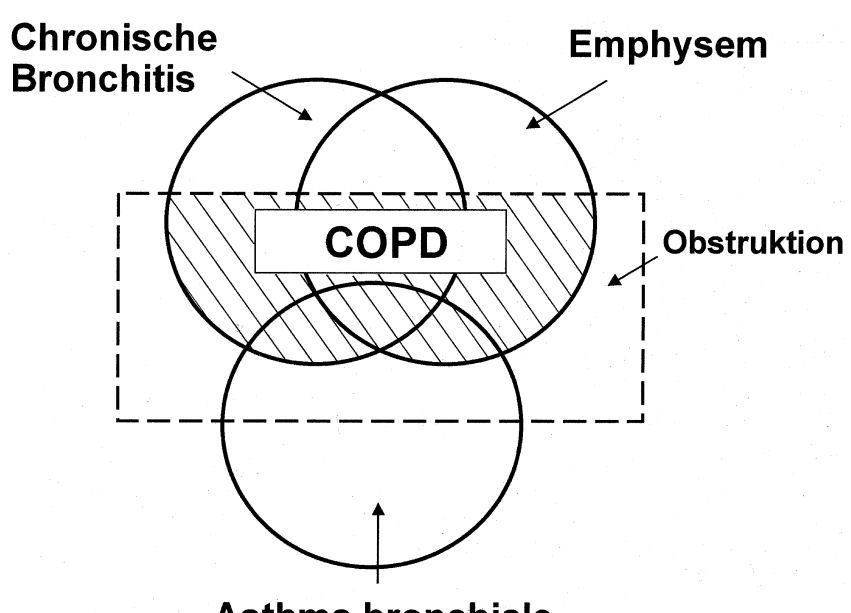

Asthma bronchiale

Abb. 1 Nichtproportionales Venn-Diagramm der COPD: Beziehung zwischen chronischer Bronchitis, Emphysem, Asthma bronchiale und Atemflußbehinderung [3]. Die chronische Bronchitis ist klinisch durch einen produktiven Husten gekennzeichnet, sie kann mit oder ohne Obstruktion vorkommen. Die beim Lungenemphysem vorhandene Parenchymdestruktion führt zu einer Verminderung der radialen Zugkräfte auf die Bronchuswand und kann dadurch eine exspiratorische Atemflußminderung verursachen. Überlappungen dieser beiden Grundkrankheiten sind möglich. Zigarettenraucher mit einer chronischen Bronchitis können durch Destruktion des angrenzenden Lungengewebes ein zentroazinäres Lungenemphysem entwickeln. Gelegentlich werden Charakteristika eines Asthma bronchiale wie bronchiale Hyperreagibilität oder akute Reversibilität angetroffen, Patienten mit einer chronischen Bronchitis oder einem Emphysem zeigen aber niemals eine vollständige Reversibilität. Die Untergruppe mit einer COPD ist schraffiert dargestellt, Patienten mit Asthma bronchiale und vollständig reversibler Atemwegsobstruktion sind ausgegrenzt.

Das zentroazinäre oder zentrolobuläre Emphysem beginnt in den Bronchioli respiratorii und dehnt sich von den zentralen Abschnitten des Azinus nach peripher aus. Die Oberlappen sind bevorzugt betroffen. Dieser Emphysemtyp entsteht durch Inhalationsnoxen, v.a. Zigarettenrauch (Abb. 2b).

Das periazinäre oder paraseptale Emphysem zeigt Veränderungen entlang von Bindegewebssepten der Lobuli und der Pleura viszeralis. Diese sind lungenfunktionell kaum bedeutsam, können aber zu einem Spontanpneumothorax führen.

Diese morphologischen Veränderungen sind schwierig nachzuweisen. Für den Kliniker wäre daher eine Definition, welche die funktionellen Auswirkungen der beim Emphysem reduzierten Gasaustauschfläche und die dadurch verminderte Diffusionskapazität berücksichtigt, geeigneter.

Findet sich beim Emphysem auch eine Obstruktion, so kommt diese durch den Parenchymverlust mit Verminderung der radialen Zugkräfte (=elastic recoil) auf die Bronchuswand zustande.

\section{Pathogenetische Modelle der Emphysementstehung}

\section{Proteasen/Antiproteasen-Modell}

Nach heutigem Wissensstand resultiert ein Lungenemphysem aus einem Ungleichgewicht zwischen proteolytischen Enzymen und antiproteolytischen Schutzmechanismen (Antiproteasen) [4]. Eine verstärkte Freisetzung von Elastase aus Neutrophilen und Makrophagen führt dabei ebenso zu einem proteolytischen Übergewicht wie ein angeborener oder erworbener Mangel an Inhibitoren (Abb. 3). Proteasen, vor allem die Elastase der Granulozyten, werden im Rahmen eines Entzündungsprozesses in den Bronchiolen und dem angrenzenden Alveolarraum freigesetzt und können Kollagen- und Elastinfasern des Lungengerüstes angreifen [55]. Daneben fördert Elastase die Produktion eines zähen Schleims, lähmt die Zilienfunktion und beeinträchtigt den Abtransport des Mucus. Dies begünstigt rezidivierende Infekte und damit wiederum eine neutrophile Entzündung. Als Schutzmechanismus dienen der $\alpha_{1}$-Proteasen-Inhibitor ( $\alpha_{1}$-Antitrypsin), der, hepatisch produziert, auf dem Blutwege überall im Körper zur Verfügung steht, sowie lokal produzierte Inhibitoren (z.B. Tissue Inhibitors of Metalloproteinases = TIMPs).

\section{Oxidantien/Antioxidantien-Balance}

Zigarettenrauch enthält reichlich reaktive Sauerstoffprodukte und wirkt in diesem Gleichgewichtssystem in mehrfacher Hinsicht deletär. Die inhalativen (exogenen) Oxidantien inaktivieren $\alpha_{1}$-Antitrypsin und steigern dadurch die Elastaseninduzierte Gewebsschädigung. Daneben aktiviert Tabakrauch Alveolarmakrophagen. Diese mononukleäre Entzündung wird als früheste Läsion bei jungen Rauchern angetroffen. Die Parenchymdestruktion scheint mit der Makrophagenzahl zu korrelieren [55]. Makrophagen sezernieren proteolytische Enzyme und chemotaktische Faktoren wie IL-8 und Leukotrien $\mathrm{B}_{4}\left(=\mathrm{LTB}_{4}\right)$. Hierdurch werden neutrophile Granulozyten angelockt. Diese setzen Proteasen wie Neutrophilen-Elastase und Metalloproteinasen sowie endogene Oxidantien frei. Oxidantien wirken direkt toxisch auf Alveolarstrukturen und hemmen die Antielastasen, welche das Lungengerüst schützen sollten. Daneben blockieren sie Reparaturmechanismen, welche die geschädigten Strukturelemente regenerieren könnten. Elastin ist die Hauptkomponente elastischer Fasern. Zigarettenrauch hemmt das Enzym Lysyloxidase, welches für die Quervernetzung neusynthetisierter Elastinvorstufen notwendig ist. Desmosine entstehen aus vernetzten Lysinresten. Sie sind Marker des Elastinabbaus. Ihre Ausscheidung im Urin von Rauchern korreliert mit der Rate des FEV $_{1}$-Abfalls $[32,59]$. Antioxidantien bieten einen Schutz vor Oxidantien, sie können enzymatischer (Superoxid-Dismutase, Katalase, Glutathion Peroxidase) oder nichtenzymatischer (Glutathion, Vitamin C, Beta-Carotin) Natur sein [51].

\section{Auslösende Faktoren}

Rauchen. Die Dynamik des jährlichen Abfalls des $\mathrm{FEV}_{1}$ ist bei Rauchern beschleunigt und hat eine entscheidende Bedeutung für den Krankheitsverlauf der COPD. Die Lung Health Study [2] hat den Einfluß des Rauchens auf das $\mathrm{FEV}_{1}$ bei Patienten mit leichter COPD ( $\mathrm{FEV}_{1} 55-90 \%$ Soll) eindrücklich belegt. Multizentrisch wurden 5887 Raucher im Alter von 35 bis 60 Jahren in 3 Gruppen randomisiert und über 5 Jahre 

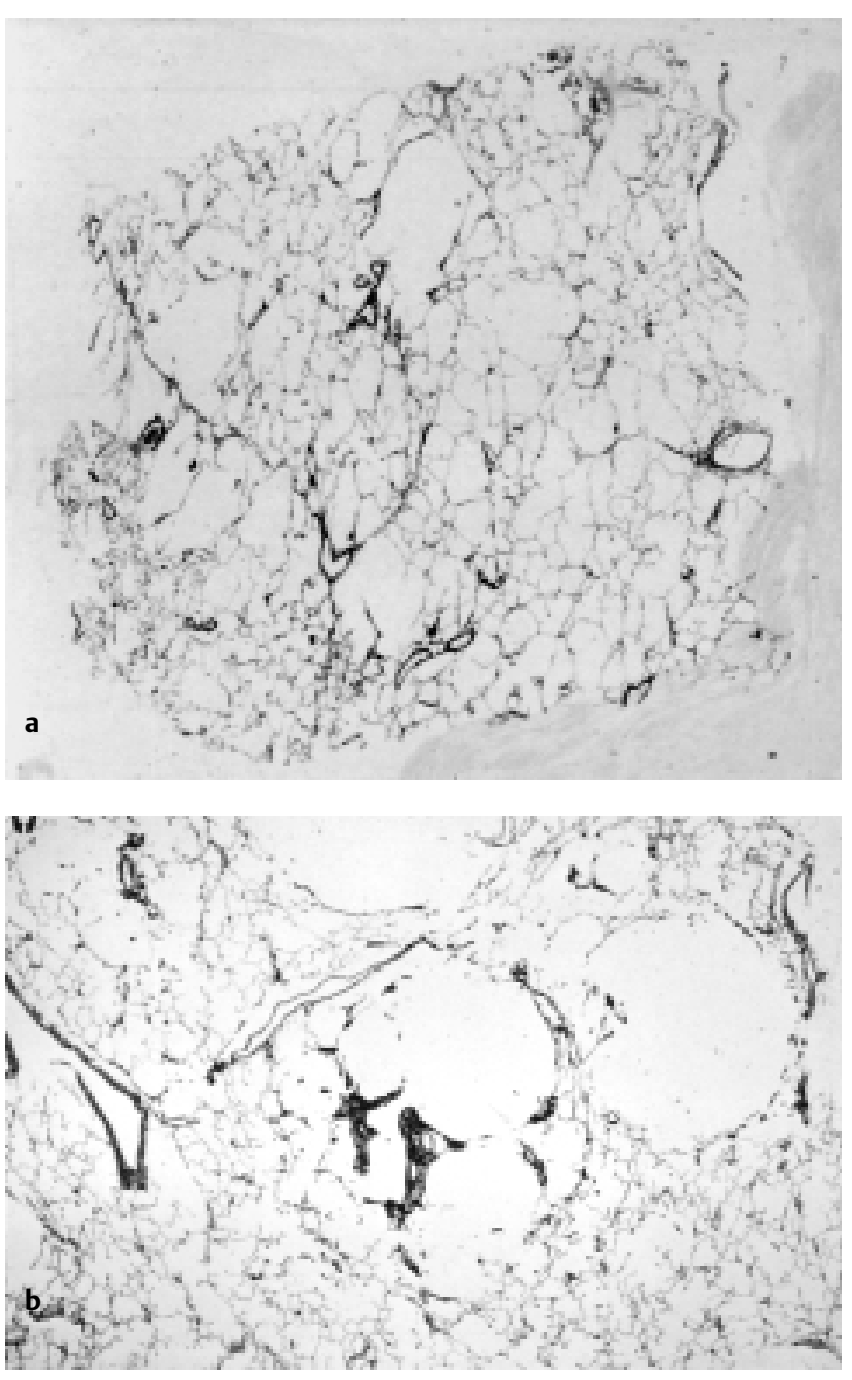

Abb. 2 Emphysemmorphologien.

2a Panazinäres Emphysem.

Die Läsionen sind diffus auf den Lobulus verteilt und vorwiegend peripher lokalisiert. Die Alveolargänge sind ausgeweitet ohne Bezug zu den kleinen Atemwegen. Sekundär schwinden die intraazinären Strukturen.

2b Zentroazinäres Emphysem.

Die Läsionen beginnen in den Bronchioli terminales und dehnen sich nach peripher aus. Die periphere Alveolarstruktur bleibt zunächst erhalten, geht aber sekundär zugrunde.

(Bilder von Prof. P. Dalquen, Pathologisches Institut des Kantonsspitals Basel).

verfolgt. Zwei Drittel wurden in ein intensives Nikotinentwöhnungsprogramm einbezogen, ein Drittel erhielt keine Intervention. Patienten, welche erfolgreich entwöhnt werden konnten und auch Nichtraucher blieben, wiesen im Vergleich $\mathrm{zu}$ persistierenden Rauchern einen signifikant geringeren Abfall des $\mathrm{FEV}_{1}$ auf (absoluter Abfall $72 \mathrm{ml}$ versus $301 \mathrm{ml}$ in 5 Jahren). Der Einfluß des Zigarettenrauchens auf die Mortalität wurde von Doll et al. in einer Langzeitstudie über 40 Jahre an 34439 britischen Ärzten untersucht [19]. Die Mortalität stieg bei Rauchern in Abhängigkeit des täglichen Zigarettenkonsums an. Während bei den 70jährigen noch $80 \%$ der Nichtraucher am Leben waren, erreichten nur 50\% der schweren Raucher (Zigarettenkonsum pro Tag $>25$ ) dieses Alter. Die

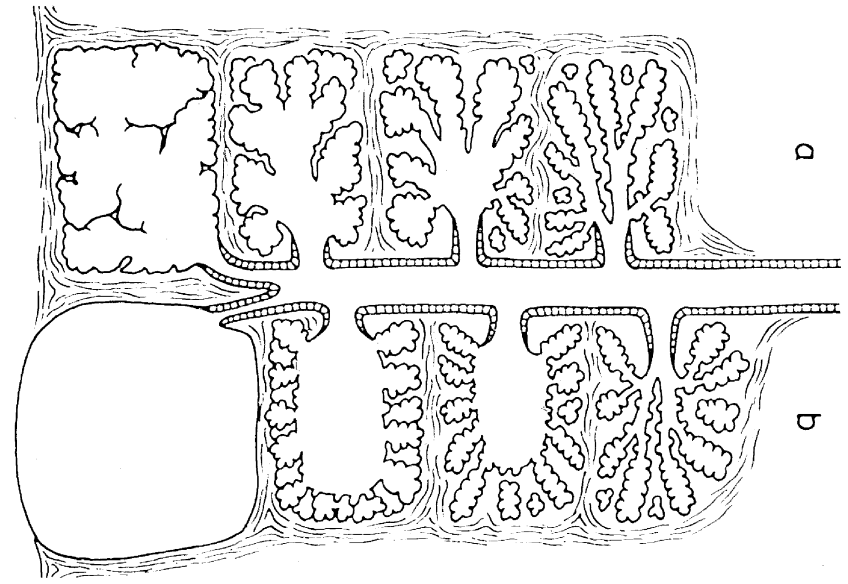

Skizze zu Abb. 2a u. b

Lebenserwartung aller Raucher lag durchschnittlich 8 Jahre niedriger als die von Nichtrauchern.

Umweltverschmutzung, Staubexposition am Arbeitsplatz und inhalative Noxen einschließlich Passivrauchen sind weitere Faktoren bei der Entwicklung einer COPD. Die Swiss Study on Air Pollution and Lung Diseases in Adults (= SAPALDIA) hat an 8 schweizerischen Zentren 4197 Nieraucher im Alter von 18 bis 60 Jahren untersucht [35]. 1259 davon waren mindestens in den vorausgehenden 12 Monaten passiv Ziga-

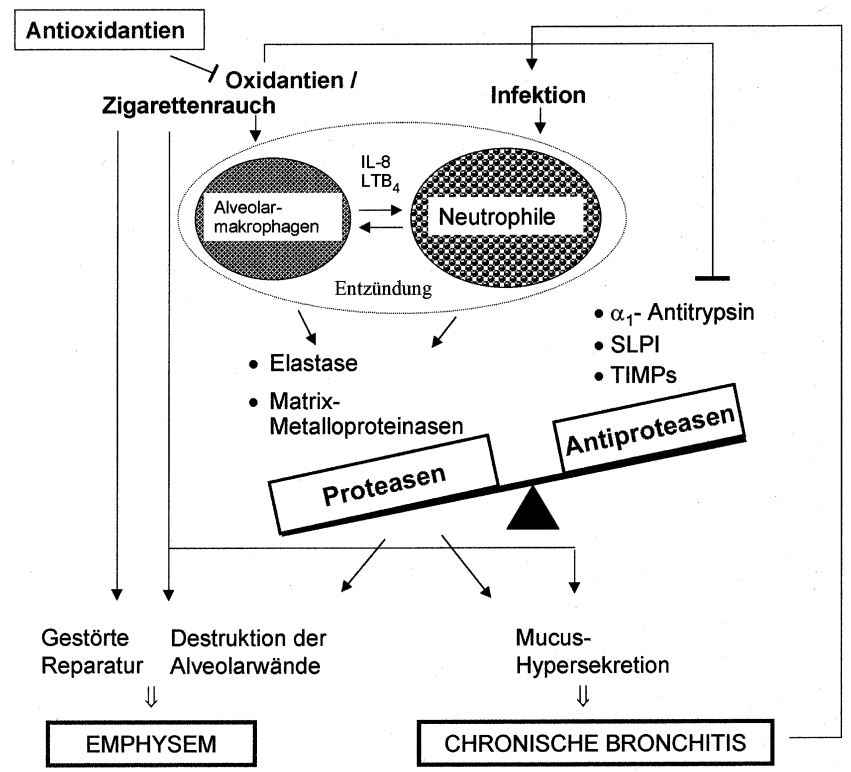

Abb. 3 Proteasen/Antiproteasen-Modell der Emphysementstehung. Ein Emphysem resultiert aus einem Ungleichgewicht proteolytischer, die Matrixstrukturen zerstörender Enzyme und antiproteolytischer Schutzmechanismen. Aktivierte Alveolarmakrophagen setzen chemotaktische Faktoren frei, welche neutrophile Granulozyten anlocken. Oxidantien steigern diese neutrophile Entzündung. Daneben wirken sie direkt gewebstoxisch und behindern die Neusynthese von Elastin. Die vermehrte Produktion eines zähen Sekrets ist das Charakteristikum der chronischen Bronchitis und begünstigt sekundäre Infekte. Antioxidantien schützen vor diesen reaktiven Sauerstoffprodukten. 
rettenrauch exponiert gewesen. Dieses Kollektiv wies im Vergleich zu den übrigen Nierauchern ein signifikant höheres Risiko für das Auftreten einer keuchenden Atmung ohne grippalen Infekt, einer Anstrengungsdyspnoe, eines vom Arzt diagnostizierten Asthma bronchiale sowie Bronchitis-Symptome auf. Während die erstgenannten Beschwerden mit der täglichen Expositionsdauer korrelierten, waren die Bronchitis-Symptome eher von der Gesamtexpositionsdauer in Jahren abhängig. Die SAPALDIA-Studie untersuchte auch die Langzeitauswirkungen mittelstarker Luftverschmutzung. Der Grad der Umgebungsbelastung wurde als Konzentration der inhalierbaren Schwebestaubfraktion mit einer Partikelgröße unter $10 \mu \mathrm{m}\left(=\mathrm{PM}_{10}\right)$ gemessen. Nieraucher in stärker belasteter Umgebung beklagten häufiger Symptome einer chronischen Bronchitis und Atemnot (prozentualer Anstieg der Symptomprävalenz bei einem Anstieg des Jahresmittelwertes von $\mathrm{PM}_{10}$ um $10 \mu \mathrm{g} / \mathrm{m}^{3}$ : chronisch produktiver Husten $35 \%$, Atemnot am Tage 47\%, Anstrengungsdyspnoe 31,6\%) [70]. $\mathrm{PM}_{10}$ bezeichnet solide und tropfenförmige Partikel mit einem mittleren medianen Durchmesser bis $10 \mu \mathrm{m}$. Die Atemluft ist zu ca. je $50 \%$ mit Partikeln unter oder über $2,5 \mu \mathrm{m}$ belastet. Partikel über 2,5 $\mu \mathrm{m}$ sind meist natürlichen Ursprungs (Bakterien, Erdstaub), während die feinen und ultrafeinen Partikel durch Verbrennung fossiler Brennstoffe (Kondensate gasförmiger Verbrennungsprodukte) entstehen. Während erstere vorwiegend bronchial abgelagert und durch den mukoziliären Apparat eliminiert werden, gelangen die feinen Partikel bis in die Alveolen, können ins Lungenparenchym eindringen und dort eine Alveolitis auslösen. Eine Zunahme des Tagesmittelwertes von $\mathrm{PM}_{10}$ um $10 \mu \mathrm{g} / \mathrm{m}^{3}$ ist mit einer Zunahme der nichtunfallbedingten Todesfälle am gleichen oder nachfolgenden Tag um 1\% korreliert. Patienten mit vorbestehender Atemwegsobstruktion sind besonders hinsichtlich einer Deposition von Partikeln und einer dadurch höheren Mortalität gefährdet [9].

5-10\% der Patienten mit COPD haben nie geraucht, andererseits entwickeln nur ca. $15 \%$ aller regelmäßigen Raucher eine COPD [62]. Die starke Variabilität des $\mathrm{FEV}_{1}$-Abfalls trotz identischen Tabakkonsums wird auf eine genetische Disposition zurückgeführt [53]. Verwandte ersten Grades von Patienten mit early-onset COPD ohne $\alpha_{1}$-Antitrypsin-Defizienz haben im Vergleich mit Kontrollpersonen niedrigere $\mathrm{FEV}_{1^{-}}$ Werte. Verwandte, welche rauchen oder Exraucher sind, haben ein 3 fach höheres Risiko, ein $\mathrm{FEV}_{1}<60 \%$ Soll oder eine chronische Bronchitis zu entwickeln [57]. Beim Tabakrauchen entstehen reaktive Epoxidmediatoren, welche von einer mikrosomalen Epoxid-Hydrolase metabolisiert werden. Für dieses Enzym ist ein Polymorphismus bekannt $[49,59]$. Individuen mit einem bestimmten Phänotyp (homozygot für das langsam aktive Enzym = slow metabolisers) haben ein 4- bis 5 fach höheres Risiko für die Entwicklung einer COPD oder eines Emphysems. Es scheint also auch eine genetische Empfindlichkeit gegenüber oxidativem Streß und nicht nur bei einem Antiproteasenmangel zu geben.

Ein $\boldsymbol{\alpha}_{\mathbf{1}}$-Antitrypsin-Mangel ist der am besten dokumentierte genetische Risikofaktor für die Entwicklung einer COPD, dieses Kollektiv macht aber nur 1\% der COPD-Patienten aus. Patienten, welche homozygot den Phänotyp Pi Z aufweisen und zusätzlich rauchen, sind besonders gefährdet. Das defiziente Protein wird nach Synthese in der Leber nur ungenügend in die Blutbahn sezerniert und hat eine geringere
Assoziationsrate zu Elastase, ist dadurch weniger effektiv und bedingt ein 30fach gesteigertes Emphysemrisiko. Bei heterozygoten Trägern des Phänotyps $\mathrm{Pi} \mathrm{Z}$ ( $\mathrm{Pi} \mathrm{MZ}$ ) liegt das Emphysemrisiko nur 1,5- bis 5fach höher. Eine Serumaktivität des $\alpha_{1}$-Antitrypsins unter $37 \%$ Soll $(<80 \mathrm{mg} / \mathrm{dl})$ weist auf ein erhöhtes Emphysemrisiko hin [55].

\section{Diagnostik}

Tab. 2 zeigt eine Übersicht charakteristischer klinischer, lungenfunktioneller und radiologischer Befunde bei Patienten mit COPD. Die Anamnese sollte frühere und aktuelle Rauchge-

Tab. 2 Klinische Charakteristika und Diagnostik der COPD

Anamnese* Inhalationsnoxen: aktueller Rauchkonsum und bisherige packyears**

Arbeitsplatz/berufliche Belastung

Familienanamnese: genetische Belastung, Passivrauchen

Dyspnoe/Leistungsfähigkeit

Sputumproduktion

Exazerbation/Infekthinweise (purulentes Sputum,

Fieber, vermehrte Dyspnoe)

Status* Zyanose, Trommelschlegelfinger/Uhrglasnägel Emphysemthorax, tiefstehende Zwerchfelle Abgeschwächtes Atemgeräusch, verlängertes Exspirium, Giemen oder Brummen

Rechtsherzbelastungszeichen

bildgebende Thoraxübersicht*: Emphysemzeichen (= regionale Verfahren Transparenzerhöhung/Rarefizierung der Gefäße als Korrelat von Emphysem-bullae, horizontal gestellt Rippen, vergrößerter Retrosternalraum, abgeflachte Zwerchfelle als Korrelat der Hyperinflation) Ausschluß von Lungeninfiltrat, Raumforderung oder Pneumothorax

CT-Thorax (auch Dünnschichten): Bullae, Emphysemausmaß und -lokalisation, zusätzliche Bronchiektasen

lungen- Ganzkörperplethysmographie* (Spirometrie zur Verfunktionelle laufsbeurteilung): TLC und RV (Lungenüberblähung) Diagnostik $\quad \mathrm{FEV}_{1} / \mathrm{FVC}$ in (Obstruktion), $\mathrm{FEV}_{1}$ (Schweregrad der Obstruktion)

Fluß/Volumenkurve: exspiratorischer Kollaps der Atemwege?

Spirometrie vor/nach Bronchodilatation: Reversibilität?

Diffusionskapazität

Belastungstests (6-Minuten-Gehtest oder Ergometrie) Blutgase: Hyperkapnie, Hypoxämie/Indikation zur Sauerstofflangzeittherapie?

Nächtliche Pulsoximetrie, Polysomnographie: Indikation für eine intermittierende Selbstbeatmung?

erweiterte EKG, Echokardiographie, Rechtsherzkatheter: RechtsDiagnostik herzbelastungszeichen, pulmonal-arterielle Hypertonie?

Blutbild: Polyzythämie als Korrelat einer chronischen Hypoxämie?

$\alpha_{1}$-Antitrypsin

Follow up Schweregrad:

$$
\text { leicht } \rightarrow \mathrm{FEV}_{1} \text {, FVC jährlich }
$$

mittel $\rightarrow$ Ganzkörperplethysmographie und ABGA

$1-2 \times$ pro Jahr

schwer $\rightarrow$ fachärztliche Kontrollen

\footnotetext{
* Unverzichtbare Basisuntersuchungen

** 1 packyear = Konsum von 20 Zigaretten täglich über 1 Jahr

(oder 10 Zigaretten täglich über 2 Jahre usw.)
} 
wohnheiten, andere inhalative Noxen und die aktuellen Beschwerden erfragen. Atemnot ist eine subjektive Empfindung und korreliert nur ungenügend mit der objektiv gemessenen Lungenfunktion. Initial sollte eine Ganzkörperplethysmographie erfolgen, zur Verlaufskontrolle genügt eine Spirometrie. Blutgasanalyse, Diffusionskapazitätsbestimmung und ein 6-Minuten-Gehtest ergänzen die Lungenfunktionsdiagnostik. Bei der klinischen Untersuchung sollte auf Zeichen einer chronischen Hypoxämie, Lungenüberblähung oder Rechtsherzbelastung geachtet werden. Das Thoraxübersichtsbild dient dem Ausschluß anderer Dyspnoeursachen, zur Beurteilung eines Emphysems ist ein CT überlegen. Weiterführende Abklärungen sind speziellen Fragestellungen vorbehalten.

\section{Therapeutische Strategien}

Ideales Therapieziel wäre eine Prävention der COPD. Prophylaktische Maßnahmen wie z.B. Nikotinentwöhnungsprogramme können die Entstehung bzw. das Fortschreiten der Erkrankung verhindern. Die Diagnose sollte frühestmöglich gestellt, Risikopatienten erkannt und regelmäßig kontrolliert werden. Bei manifester COPD zielen die therapeutischen Bemühungen auf eine Reduktion von Symptomen und Exazerbationen, die Erhaltung einer optimalen Lungenfunktion sowie eine Steigerung von Lebensqualität und Leistungsfähigkeit ab. Von zahlreichen Arbeitsgruppen wurden Therapierichtlinien erarbeitet $[3,11,34,56,68]$.

\section{Prophylaktische Maßnahmen}

Nikotinentwöhnung ist die einzig dokumentierte Maßnahme, um das Fortschreiten der COPD zu beeinflussen [2]. Das $\mathrm{FEV}_{1}$ eines Nichtrauchers vermindert sich nach dem 25. Lebensjahr um etwa 20 bis $30 \mathrm{ml}$ pro Jahr, beim Raucher sind dies 50 bis $60 \mathrm{ml}$ jährlich. Etwa 15\% aller Raucher zeigen einen akzelerierten Abfall des $\mathrm{FEV}_{1}$. Sie sind besonders für die Entwicklung einer COPD gefährdet. Ein Exraucher gewinnt das eingebüßte dynamische Lungenvolumen nicht mehr zurück, erreicht aber wieder den Abfall eines Nichtrauchers von ca. $20 \mathrm{ml}$ jährlich.

Die meisten erwachsenen regelmäßigen Raucher würden gerne von ihrer Sucht loskommen. Ohne unterstützende Maßnahmen gelingt dies nur ca. 2\%, nach einer kurzen ärztlichen Beratung ca. 5\%. Nikotinersatzverfahren wie Nikotinpflaster oder -nasenspray steigern die Erfolgsraten auf ca. $10 \%$ [22]. Umfassende Programme, d.h. Nikotinersatzverfahren ergänzt durch eine psychologische Betreuung, konnten in den USA ca. 30-40\% der regelmäßigen Raucher langfristig entwöhnen [2]. Ein neues Antidepressivum (Bupropion) wurde kürzlich erfolgreich zur Raucherentwöhnung eingesetzt. Während nach 7 Wochen in der Plazebo-Gruppe nur 19\% Nichtraucher geworden waren, hatten in der BupropionGruppe $44 \%$ aufgehört zu rauchen [25].

Impfungen. Prophylaktisch werden jährliche Grippeimpfungen [21] sowie von einigen Arbeitsgruppen eine einmalige Pneumokokkenimpfung [23] empfohlen. Während sich jährliche Grippeimpfungen bei über 65jährigen Patienten mit chronischen Lungenerkrankungen als effektiv und kostengünstig erwiesen haben und daher in die wichtigsten internationalen Therapierichtlinien aufgenommen wurden, ist der Benefit einer Pneumokokkenimpfung weniger gut belegt. Die amerikanischen und schweizerischen Therapierichtlinien $[3,34]$ empfehlen eine Pneumokokkenimpfung, während die europäischen und britischen Guidelines $[11,56]$ weitere Studien zur Wirksamkeit fordern.

Antioxidantien [51] $\mathrm{H}_{2} \mathrm{O}_{2}$ und $\mathrm{NO}$ in der Ausatemluft gelten als Indikatoren für oxidativen Streß, ihre Konzentrationen sind bei Patienten mit exazerbierter oder fortgeschrittener COPD sowie nach akuter Inhalation von Zigarettenrauch erhöht $[15,40]$. Das schwefelhaltige Antioxidans N-Acetylcystein (NAC) wurde ursprünglich als Mucolytikum eingesetzt. Es wirkt aber auch antioxidativ und kann Cystein für die Synthese des wichtigsten Redoxsystems Glutathion bereitstellen. In klinischen Studien wurde unter der Einnahme von NAC eine Abnahme von Virusinfekten, Exazerbationen und jährlichem $\mathrm{FEV}_{1}$-Abfall beobachtet [38]. Bei Patienten mit rezidivierenden Exazerbationen könnte daher eine prophylaktische Einnahme von NAC, insbesondere während der Wintermonate, nützlich sein.

\section{Inhalative Bronchodilatatoren}

Anticholinergika blockieren durch Hemmung der vagalen Stimulation des Bronchialbaums die Kontraktion glatter Muskelzellen und die Sekretion der Bronchialdrüsen. Im Anfall sind sie potente Bronchodilatatoren. Sie wirken bronchoselektiv bei Inhalation, haben keine systemischen Nebenwirkungen und führen nicht zu einer Toleranzentwicklung. Mit $\beta_{2^{-}}$ Mimetika wirken sie synergistisch [17]. Anticholinergika waren bisher Mittel der Wahl bei COPD. Sie scheinen kurzwirksamen $\beta_{2}$-Agonisten überlegen [50]. Eine dreimonatige Therapie mit Ipratropiumbromid führte bei Patienten mit COPD zu einer Verbesserung von $\mathrm{FEV}_{1}$ und FVC, während $\beta_{2^{-}}$ Agonisten keine signifikante Änderung bewirkten. Die akute Reversibilität nahm unter letzteren sogar ab. Im Vergleich mit langwirksamen $\beta_{2}$-Agonisten war Ipratropiumbromid im Hinblick auf die lungenfunktionelle Verbesserung jedoch nicht überlegen. Salmeterol mußte aufgrund der längeren Wirkdauer nur halb so häufig inhaliert werden und wurde daher als vorteilhafter eingestuft [1].

Neue Kenntnisse der Muscarin-Rezeptorsubtypen haben die Entwicklung selektiver Anticholinergika ermöglicht. Die bronchokonstriktorische Wirkung von Acetylcholin wird über $\mathrm{M}_{3}$ Rezeptoren der Atemwege vermittelt. $\mathrm{M}_{1}$-Rezeptoren parasympathischer Ganglien bedingen eine reflektorische Bronchokonstriktion. Blockade dieser beiden Rezeptoren erzeugt daher eine Bronchodilatation. $\mathrm{M}_{2}$-Rezeptoren cholinerger Nervenendigungen dagegen hemmen die Freisetzung von Acetylcholin. Blockade dieser Rezeptoren erhöht die Acetylcholinfreisetzung und mindert dadurch den angestrebten bronchodilatatorischen Effekt. Tiotropiumbromid ist ein neues, kinetisch selektives Anticholinergikum, welches schnell vom $\mathrm{M}_{2}$-Rezeptor, jedoch nur langsam von $\mathrm{M}_{1}$ - und $\mathrm{M}_{3}$ Rezeptoren dissoziiert. Erste klinische Studien belegen eine gute Dosis-Wirkungs-Beziehung. Tiotropiumbromid führt bei COPD-Patienten nach Inhalation einer Einzeldosis zu einem raschen Anstieg des $\mathrm{FEV}_{1}$ um durchschnittlich $300 \mathrm{ml}$ und erreicht nach 32 Stunden im Vergleich zum Basiswert noch immer eine durchschnittliche $\mathrm{FEV}_{1}$-Steigerung um $150 \mathrm{ml}$ [39]. 
Die Langzeitwirkung von Anticholinergika wurde in der Lung Health Study [2] untersucht. Raucher mit COPD wurden einem Nikotinentwöhnungsprogramm unterzogen und inhalierten zusätzlich entweder ein Anticholinergikum (Ipratropiumbromid $3 \times 2$ Hübe tgl.) oder Plazebo. Die regelmäßige Inhalation von Ipratropiumbromid führte zu einem Anstieg des $\mathrm{FEV}_{1} \mathrm{um}$ $27 \mathrm{ml}$. Trotz 5jähriger Therapiedauer klang diese Verbesserung nach Absetzen des Anticholinergikums innerhalb von 40 Stunden ab. Auch andere Studien belegen, daß bei Patienten mit COPD nur eine Nikotinabstinenz das Fortschreiten der Erkrankung aufhalten kann, während inhalative Bronchodilatatoren lediglich symptomatisch wirksam sind.

Die Wirkungen von $\boldsymbol{\beta}_{\mathbf{2}}$-Mimetika umfassen eine direkte Dilatation der Bronchien, eine Verbesserung der mukoziliären Clearance sowie eine Abnahme der dynamischen Überblähung. Als unerwünschte Folgen können ein Tremor, eine Tachykardie und eine pulmonale Vasodilatation auftreten. Zudem kommt es akut oft zu einem Mismatch von Ventilation und Perfusion mit konsekutivem, leichtem Abfall der Sauerstoffspannung. Kurzwirksame $\beta_{2}$-Mimetika werden aufgrund ihres raschen Wirkungseintritts als Rescue-Medikation bei Atemnot eingesetzt. Auch bei lungenfunktionell fehlender akuter Reversibilität können Bronchodilatatoren mittelfristig zu einer Symptombesserung führen. Durch Verminderung der Hyperinflation kann die Belastungsdyspnoe reduziert und die Belastbarkeit gesteigert werden [5].

Langwirksame inhalative $\beta_{2}$-Agonisten (Salmeterol und Formoterol) eignen sich zur Erhaltungstherapie. In einer Plazebokontrollierten Studie an Patienten mit nicht-reversibler COPD konnte die Inhalation von $50 \mu \mathrm{g}$ Salmeterol täglich die respiratorischen Beschwerden am Tage sowie das Auftreten nächtlicher Dyspnoeattacken mindern und den Bedarf an zusätzlichem Salbutamol senken. Das $\mathrm{FEV}_{1}$ stieg in den 16 Behandlungswochen um bis zu 7\% an. Nach einem 6-Minuten-Gehtest war die Kurzatmigkeit geringer als zuvor, die Gehstrecke war allerdings nicht signifikant länger [8]. Eine Arbeitsgruppe [29] konnte zeigen, daß die Inhalation von Salmeterol $50 \mu \mathrm{g}$ zweimal täglich die anhand von Fragebogen ermittelte Lebensqualität steigern kann. Gleichzeitig manifestierte sich ein leichter, jedoch signifikanter Anstieg des $\mathrm{FEV}_{1}$. Sie folgerte daher, daß schon eine moderate Verbesserung der Lungenfunktion mit einem klinisch signifikanten Gewinn an Gesundheit und Wohlbefinden verbunden sein kann. Die doppelte Salmeteroldosis erzielte keine weitere Verbesserung, war aber durch mehr Nebenwirkungen (Tremor) belastet [8]. Die regelmäßige Anwendung eines langwirksamen $\beta_{2}$-Mimetikums schmälert die bronchodilatatorische Wirkung eines zusätzlichen kurzwirksamen $\beta_{2}$-Mimetikums nicht, eine geringere Rezeptorempfindlichkeit bei Dauermedikation muß nicht befürchtet werden [33].

\section{Steroide}

Inhalative Steroide bilden die antiinflammatorische Basistherapie des Asthma bronchiale. Bei Patienten mit COPD dagegen ist ihr Einsatz umstritten. Die meisten Richtlinien empfehlen bei Patienten mit COPD einen Steroid-Trial, um den Steroideffekt zu beurteilen (Abb. 4 [3,34,68]). Dabei werden zwei Wochen lang $40 \mathrm{mg}$ Prednisolon täglich per os verabreicht. Ein Anstieg des $\mathrm{FEV}_{1}$ um mindestens $15 \%$ mit einem absoluten Anstieg um mindestens $200 \mathrm{ml}$ nach dieser Zeit wird als erfolgreiches Ansprechen gewertet. Etwa 10-15\% der Patienten sind Responders. Es wird angenommen, daß dieses Kollektiv Asthmakomponenten aufweist (vgl. Abb. 1). Chanez [16] bronchoskopierte COPD-Patienten, welche auf die Inhalation von Salbutamol keine akute Reversibilität gezeigt hatten, und unterzog sie danach einem Steroidversuch. Die SteroidReversibilität konnte mit Asthma-Charakteristika korreliert werden: Responders unterschieden sich durch signifikant höhere Eosinophilenzahlen in der BAL sowie dickere Basalmembranen in der Schleimhautbiopsie.

Sollen Non-Responders inhalative Steroide bekommen? Ein Kurzzeiteffekt fehlt sicherlich. Haben Steroide einen Effekt auf die neutrophile Entzündung bei COPD? Keatings [30] fand nach Applikation oraler oder inhalativer Steroide über 2 Wochen keinen Einfluß auf die Entzündungsparameter bei COPD-Patienten. Eine andere Studie [18] dagegen ermittelte unter hochdosierten inhalativen Steroiden nach 2 Monaten eine Reduktion der Neutrophilen- und Gesamtzellzahl im induzierten Sputum. Bereits Jones [37] hatte nach topischen Steroiden eine verminderte chemotaktische Aktivität (als Korrelat einer geringeren Rekrutierung von Neutrophilen) und eine Erhöhung der Neutrophilen-Elastase-Inhibitoren im Sputum gefunden. Können Steroide eventuell langfristig durch Verminderung der neutrophilen Entzündung einen Benefit erreichen? Eine Arbeitsgruppe [7] hat Non-Responders mit mittelschwerer COPD über 6 Monate mit inhalativen Steroiden versus Plazebo behandelt. $\mathrm{FEV}_{1}$ und klinische Symptomatik unterschieden sich danach nicht signifikant, es war weder ein physiologischer noch funktioneller Benefit vorhanden. Andere Untersucher [48] fanden nach Verabreichung topischer Steroide über 2 Jahre eine Verminderung der pulmonalen Beschwerden und eine (nichtsignifikante) Tendenz zu einem geringeren $\mathrm{FEV}_{1}$-Abfall. Neuere Studien haben klinische Parameter gewählt. Paggiaro et al. [43] behandelten COPD-Patienten über 6 Monate mit hochdosiertem inhalativen Fluticason oder Plazebo. Die Steroidgruppe hatte weniger schwere Exazerbationen, einen leichten, aber signifikanten Anstieg von $\mathrm{FEV}_{1}$, FVC und Peak flow sowie der Gehstrecke im 6-Minuten-Gehversuch.

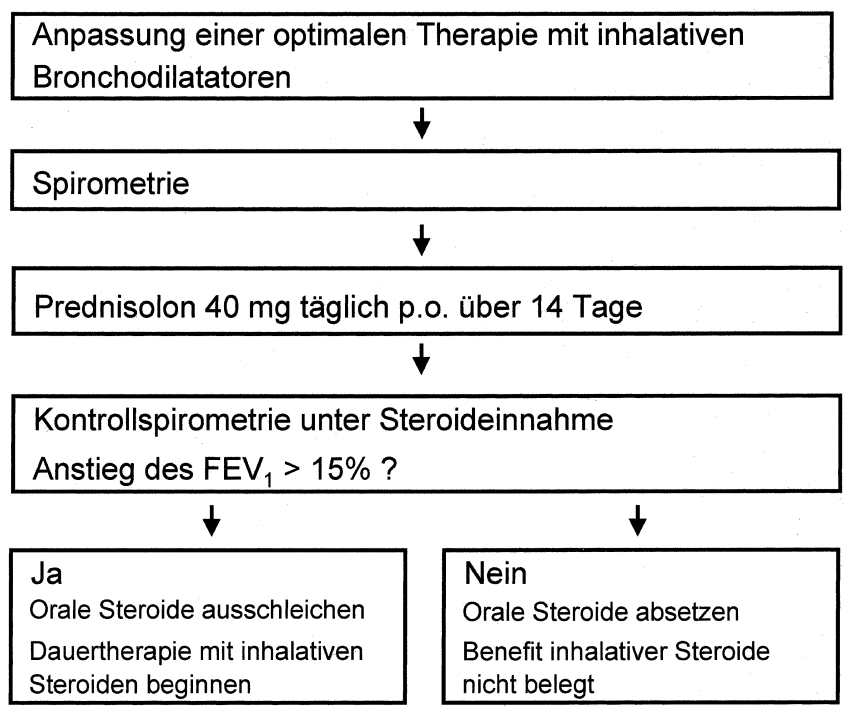

Abb. 4 Steroid-Trial. 
Drei plazebokontrollierte, randomisierte und doppelblinde Studien haben es sich zum Ziel gemacht, den Langzeiteffekt inhalativer Steroide bei COPD-Patienten $\mathrm{zu}$ untersuchen. In der multizentrischen EUROSCOP-Studie inhalierten 1277 Raucher im Alter von 30 bis 65 Jahren mit leichter COPD (mittleres $\mathrm{FEV}_{1} 77 \%$ Soll) drei Jahre lang entweder $800 \mu \mathrm{g}$ Budesonid oder Plazebo. Zwar verbesserte sich das $\mathrm{FEV}_{1}$ unter Steroidtherapie im ersten Halbjahr signifikant (Rate des $\mathrm{FEV}_{1}+17$ versus $\left.-81 \mathrm{ml} / \mathrm{Jahr}, \mathrm{p}<0,001\right)$, danach aber war der jährliche Abfall des $\mathrm{FEV}_{1}$ in beiden Gruppen identisch (- 57 vs. $-69 \mathrm{ml} / \mathrm{Jahr}, \mathrm{p}=0,39)$. Der Benefit einer regelmäßigen Inhalation von Steroiden war somit einmalig und auf die ersten Therapiemonate beschränkt, der Langzeitverlauf des $\mathrm{FEV}_{1}$ konnte nicht beeinflußt werden [44]. Auch die Copenhagen City Heart Study untersuchte den jährlichen FEV $_{1}$-Abfall unter Inhalation topischer Steroide (Budesonid 1,2 g täglich in den ersten 6 Monaten, dann $800 \mu \mathrm{g}$ täglich über weitere $2 \frac{1}{2}$ Jahre) oder Plazebo. Die 290 Patienten mit leichter COPD (mittleres $\mathrm{FEV}_{1} 86 \%$ Soll) waren durchschnittlich 59 Jahre alt. Einen signifikanten oder klinisch relevanten Langzeiteffekt konnten auch diese Arbeitsgruppen nicht finden (Abfall des $\mathrm{FEV}_{1}$ in der Budesonid-Gruppe - 45,1 ml/Jahr versus - 41,8 ml/ Jahr in der Plazebo-Gruppe) [67]. In der ISOLDE-Studie inhalierten Patienten mit schwerer COPD (mittleres FEV $_{1} 50 \%$ Soll) drei Jahre lang entweder $1 \mathrm{mg}$ Fluticason täglich oder Plazebo. Die Resultate wurden bisher nur mündlich an Kongressen vorgestellt, eine Reduktion des $\mathrm{FEV}_{1}$-Abfalls konnten aber auch diese Arbeitsgruppen nicht belegen. Der Wegfall inhalativer Steroide in der Vorphase der Studie steigerte allerdings Auftreten von Exazerbationen und auch die Lebensqualität war unter Steroidtherapie besser [14].

Unbestritten ist der Stellenwert von Steroiden in der Therapie akuter Exazerbationen [63]. Eine ambulante systemische Steroidtherapie bei Patienten mit exazerbierter COPD (Prednisolon p.o. jeweils 3 Tage 60, 40 bzw. $20 \mathrm{mg}$ ) versus Plazebo erzielte eine raschere Verbesserung von Obstruktionsgrad, Hypoxämie und Dyspnoe. Ziel sollte es immer sein, die Steroide baldmöglichst auszuschleichen. Eine längerfristige Gabe systemischer Steroide muß im Hinblick auf den nicht eindeutig belegten Benefit und die multiplen Nebenwirkungen kritisch beurteilt werden [41,42]. Bei Respondern wird eine Umstellung auf inhalative Steroide empfohlen.

\section{Theophyllin}

Theophyllin erhöht über eine Hemmung der Phosphodiesterase das intrazelluläre cAMP. Neben einer Bronchodilatation und einer verbesserten mukoziliären Clearance sind in letzter Zeit weitere Wirkungen aufgedeckt worden. Eine Steigerung von Atemantrieb und Atemmuskelleistung, eine Erhöhung des Cardiac output sowie eine pulmonale Vasodilatation können sich bei COPD-Patienten mit Cor pulmonale günstig erweisen. Daneben wurde eine Hemmung der Neutrophilenfunktion, d.h. ein antiinflammatorischer Effekt beschrieben [47]. Bei Patienten mit Asthma bronchiale war eine Kombination von Theophyllin mit inhalativen Steroiden gleich wirksam wie eine Verdoppelung der Steroiddosis [64], entsprechende Studien mit COPD-Patienten liegen nicht vor. Die Deutsche Gesellschaft für Pneumologie empfiehlt einen Auslaßversuch in einer stabilen Krankheitsphase, um den klinischen Effekt von Theophyllin zu beurteilen. Ca. $10 \%$ sind Responders [68]. Häufig werden Tremor, Palpitationen und gastrointestinale Beschwerden beklagt. Um solche Nebenwirkungen und Serumspiegelkontrollen zu vermeiden, werden heute bewußt niedrige Dosen (400-600 mg täglich) in Retardform verabreicht. Zu beachten ist, daß Alkohol, Nikotin und verschiedene Medikamente einen gesteigerten Metabolismus in der Leber induzieren. Raucher benötigen bis zu $50 \%$ höhere Dosen. Theophyllin ist aber kein Mittel der ersten Wahl und sollte bei COPD-Patienten nur in Kombination zur Langzeittherapie eingesetzt werden $[3,34]$.

\section{Antibiotika}

Vermehrtes oder purulentes Sputum und vermehrte Dyspnoe gelten bei COPD-Patienten als klinische Zeichen einer Infektexazerbation. Oft sind Viren, daneben aber auch Infektionen mit Hämophilus influenzae, Streptococcus pneumoniae oder Branhamella catarrhalis auslösend. Finden sich zwei der oben genannten Symptome, ist eine empirische Antibiotikatherapie über 7 bis 14 Tage sinnvoll. Diese kann Schweregrad und Dauer der Exazerbation mindern. Aminopenicilline mit Betalaktamaseinhibitoren, Gyrasehemmer, Makrolide und Cephalosporine der 2. und 3. Generation finden Anwendung. Zunehmende Resistenzentwicklungen wie z.B. penicillinresistente Pneumokokken sind problematisch [69].

\section{Zukünftige medikamentöse Therapiestrategien}

Aufgrund besserer Kenntnis der pathophysiologischen und biochemischen Mechanismen zielen zahlreiche Forschungsprojekte auf die Entwicklung neuer antiinflammatorischer Substanzen. LTB4-Antagonisten, Syntheseinhibitoren und Rezeptorantagonisten von IL-8, Inhibitoren von TNF $\alpha$ und Adhäsionsmolekülen sowie Antioxidantien sollen die in Abb. 3 skizzierte neutrophile Entzündung verhindern. Synthetische Antiproteasen (Inhibitoren von Neutrophilen-Elastase, Cathepsin und Matrix-Metalloproteinasen) sowie rekombinantes, humanes $\alpha_{1}$-Antitrypsin und sekretorische Leukoproteaseinhibitoren sollen das Proteasen/Antiproteasen-Ungleichgewicht korrigieren [4].

\section{Langzeitsauerstofftherapie}

Neben der Nikotinentwöhnung ist die Sauerstofflangzeittherapie die einzig dokumentierte Therapieform, welche bei Patienten mit COPD und chronischer Hypoxämie eine Lebensverlängerung erzielen kann, sofern die tägliche Anwendungsdauer mindestens 15 Stunden beträgt. Durch eine Verbesserung der alveolären Oxygenierung wird die pulmonale Hypertonie bei diesem Patientenkollektiv vermindert und einem Rechtsherzversagen entgegengewirkt. Daneben trägt die Beseitigung der arteriellen Hypoxämie zu einer Verbesserung der psychischen und neuropsychischen Leistungsfähigkeit und damit zur Steigerung der Lebensqualität bei [71]. Als Indikation zur Langzeitsauerstofftherapie wird bei stabiler Krankheit ein Sauerstoffpartialdruck in Ruhe und bei Zimmerluft unter $55 \mathrm{~mm} \mathrm{Hg}$ angesehen [68]. Finden sich bereits Zeichen eines chronischen Cor pulmonale oder einer sekundären Polyglobulie, so sollte auch ein Sauerstoffpartialdruck unter $60 \mathrm{~mm} \mathrm{Hg}$ korrigiert werden. Ziel ist es, durch möglichst kontinuierliche Zufuhr den Sauerstoffpartialdruck auf mindestens $65 \mathrm{~mm} \mathrm{Hg}$ bzw. die Sauerstoffsättigung auf über 90\% anzuheben. Bei mobilen Patienten sollen diese Werte unter leichten Alltagsbelastungen gehalten werden und auch 
im Schlaf nicht unter diese Grenze abfallen. Eine leichte Hyperkapnie stellt keine Kontraindikation dar, bei einer (nächtlichen) $\mathrm{CO}_{2}$-Retention über $50 \mathrm{~mm} \mathrm{Hg}$ ist jedoch eine intermittierende Selbstbeatmung (= ISB) zu diskutieren [31]. Als Sauerstoffquellen kommen ein Sauerstoffkonzentrator oder, insbesondere bei mobilen Patienten, Flüssigsauerstoffreservoirs in Frage. Der Sauerstoff wird meist mittels doppellumiger Nasensonden, eventuell ergänzt durch sauerstoffsparende Ventile, appliziert. Transtracheale Katheter sind bezüglich Installation und Handhabung aufwendiger, im Hinblick auf den um ca. 50\% geringeren Sauerstoffverbrauch, die verminderte Totraumventilation und das kosmetische Resultat aber vorteilhafter [46]. Bei nächtlichen Entsättigungen muß polysomnographisch ein eventuell zusätzlich vorhandenes obstruktives Schlafapnoe-Syndrom abgegrenzt werden.

\section{Nichtinvasive Beatmung}

COPD-Patienten mit einer respiratorischen Globalinsuffizienz am Tag weisen zusätzlich nächtliche Desaturierungen auf. Im REM-Schlaf ist der Muskeltonus stark erniedrigt, die Leistung insbesondere der Atemhilfsmuskulatur entsprechend vermindert, so daß bevorzugt in diesem Schlafstadium schwere Entsättigungen auftreten können. Alleinige Sauerstoffzufuhr verstärkt die Hyperkapnie weiter, da der hypoxievermittelte Atemantrieb wegfällt. Allerdings scheint dieser bei der Langzeitsauerstofftherapie beobachtete Effekt das Überleben zu verlängern, wahrscheinlich durch die sekundäre alveoläre Hypoventilation und Schonung der Atempumpe. Therapeutisch kommt eine nächtliche nichtinvasive Beatmung mit einem Bilevel PAP-(BiPAP = bilevelpositive airway pressure) oder einem nIPPV-Ventilator (nIPPV = nasal intermittent positive pressure ventilation) in Frage. Mit einem nIPPV-Gerät kann ein höherer Inspirationsdruck aufgebaut werden. Durch eine kontrollierte intermittierende Selbstbeatmung kann die Inspirationsmuskulatur phasenweise ruhig gestellt, die erschöpfte Atemmuskulatur somit entlastet und die Hyperkapnie normalisiert werden $[24,58,45]$. Unter dieser Therapie verbessern sich Blutgase und Lebensqualität, der Langzeiteffekt wird aber noch untersucht. Nichtinvasive Beatmungsformen werden vorübergehend auch bei akuter respiratorischer Insuffizienz im Rahmen von COPD-Exazerbationen eingesetzt. Häufig kann damit eine Intubation umgangen werden [12].

\section{Pulmonale Rehabilitation}

Ein umfassendes Rehabilitationsprogramm beinhaltet eine Optimierung des Therapiemanagements im Hinblick auf Medikation und Ernährung sowie die Schulung von Patient und Angehörigen. Durch körperliches Training können maximale Übungskapazität, Gehstrecke im 6-Minuten-Gehtest und Ausdauer gesteigert werden. Die Lebensqualität nimmt zu, die Symptomatik ab [13,36]. Der Einfluß auf die Mortalität ist unklar. Eine Belastung von ca. 70\% der maximalen Arbeitsbelastung ist einem Training mit niedriger Intensität überlegen und kann den Laktatspiegel und die erforderliche Ventilation bei identischer submaximaler Belastung senken. Ein Training der Beinmuskulatur kann die Belastbarkeit am effektivsten verbessern, ein Training der Atemmuskulatur dagegen ist nicht sinnvoll, da diese bereits überbeansprucht wird. Rehabilitationsprogramme können stationär oder am- bulant erfolgen, zur Aufrechterhaltung des Trainingseffekts ist eine Fortführung zu Hause unerläßlich [20].

\section{Chirurgische Interventionen bei Lungenemphysem}

Chirurgische Therapiestrategien bei COPD oder von Komplikationen bei vorbestehender COPD umfassen Pleurodese, Bullektomie, Lungenvolumenreduktion und Lungentransplantation [6]. Die thorakoskopische Pleurodese mit Pleurektomie findet Anwendung bei sekundärem Pneumothorax nach Ruptur subpleural gelegener Bullae und kann die Rezidivrate von bis zu 50\% auf 5\% mindern. Große Emphysembullae können benachbarte Strukturen verdrängen und gesundes Lungengewebe komprimieren. Nehmen sie mehr als ein Drittel des Volumens eines Hemithorax ein, besteht die Indikation zur chirurgischen Bullaresektion.

Die Lungenüberblähung beim Emphysem führt zu einer Muskelverkürzung und ungünstigeren Zugrichtung der abgeflachten Zwerchfelle mit nachfolgend weniger effizienten Muskelkontraktionen. Die Reduktion des Lungenparenchyms begünstigt durch eine Verminderung der radialen Zugkräfte auf die Bronchuswand einen exspiratorischen Bronchialkollaps. Durch eine Lungenvolumenreduktion können die Überblähung reduziert, die Obstruktion vermindert und die Atemmechanik verbessert werden [54,61]. Patienten mit stark eingeschränkter Leistungsfähigkeit oder Ruhedyspnoe und schwerer Bronchoobstruktion mit Überblähung ohne Besserung unter konventioneller Therapie kommen für diesen Eingriff in Frage (Gehstrecke im 6-Minuten-Gehtest unter $600 \mathrm{~m}$, Totale Lungenkapazität > 125\% Soll, Residualvolumen $>200 \%$ Soll, $\mathrm{FEV}_{1}<35 \%$ Soll). Eine Hyperkapnie, pulmonale Hypertension, schwerst reduzierte Diffusionskapazität oder Bronchiektasen stellen Kontraindikationen dar (PAP mean $>35 \mathrm{~mm} \mathrm{Hg}, \mathrm{PaCO}_{2}>55 \mathrm{~mm} \mathrm{Hg}$, TLCO SB <20\% Soll). Eine schwere Systemerkrankung oder Dysfunktion anderer Organe sowie ein Alter über 75 Jahre sind ebenfalls Ausschlußkriterien. Mittels medianer Sternotomie oder videoassistierter Thorakoskopie (VATS) werden 20 bis 30\% der am meisten zerstörten Areale entfernt [52]. Bilaterale Resektionen erzielen bessere Ergebnisse als einseitige Reduktionen, eine inhomogene Emphysemverteilung ist vorteilhaft. Patienten mit präoperativ weniger stark erhöhten inspiratorischen Atemwegswiderständen erzielen postoperativ signifikant bessere Volumenzunahmen des $\mathrm{FEV}_{1}$ [26]. Hauptkomplikationen sind persistierende Luftlecks und Pneumonien. Die perioperative Mortalität liegt um 5\%. Die Funktionsverbesserung (Anstieg von $\mathrm{FEV}_{1}$ und Gehstrecke) wird rasch erreicht und scheint mindestens 12 bis 18 Monate anzuhalten.

Eine Lungentransplantation kommt bei Patienten mit Endstadium eines Lungenemphysems in Frage, sofern eine schwerste, trotz Ausschöpfung aller medikamentöser Möglichkeiten invalidisierende Dyspnoe mit einem $\mathrm{FEV}_{1}$ unter $20 \%$ vorliegt und eine Lungenvolumenreduktion nicht durchgeführt werden kann. Eine homogene Emphysemverteilung, eine pulmonalarterielle Hypertonie, Hyperkapnie oder Bronchiektasen stellen bei diesem Verfahren keine Ausschlußkriterien dar, die Alterslimite liegt aber um ca. 10 Jahre tiefer als bei der Lungenvolumenreduktion [60]. 


\section{Literatur}

${ }^{1}$ Anderson W, Wisniewski M, Barbee R, Rickard KA. Comparison of Serevent and ipratropium on pulmonary function in COPD patients reversible to ipratropium. Am J Respir Crit Care Med 1997; 155: A277

2 Anthonisen NR, Connett JE, Kiley JP, Altose MD, Bailey WD et al. Effects of Smoking Intervention and the Use of an Inhaled Anticholinergic Bronchodilatator on the Rate of Decline of $\mathrm{FEV}_{1}$. The Lung Health Study. JAMA 1994; 272: 1497 - 1505

${ }^{3}$ ATS Statement. Standards for the diagnosis and care of patients with chronic obstructive pulmonary disease. Am J Respir Crit Care Med 1995; 152: S77 - 120

${ }^{4}$ Barnes PJ. New therapies for chronic obstructive pulmonary disease. Thorax 1998; 53: 137 - 147

${ }^{5}$ Belman MJ, Botnick WC, Shin JW. Inhaled bronchodilators reduce dynamic hyperinflation during exercise in patients with chronic obstructive pulmonary disease. Am J Respir Crit Care Med 1996; 153: 967 - 975

${ }^{6}$ Bloch KE. Lungenemphysem: Indikation zur chirurgischen Behandlung. Schweiz Med Wochenschr 1998; 128: 442 - 450

${ }^{7}$ Bourbeau J, Rouleau MY, Boucher S. Randomised controlled trial of inhaled corticosteroids in patients with chronic obstructive pulmonary disease. Thorax 1998; 53: $477-482$

${ }^{8}$ Boyd G, Morice AH, Pounsford JC, Siebert M, Peslis N, Crawford C. An evaluation of salmeterol in the treatment of chronic obstructive pulmonary disease (COPD). Eur Respir J 1997; 10: $815-821$

${ }^{9}$ Brändli O. Sind inhalierte Staubpartikel schädlich für unsere Lungen? Schweiz Med Wochenschr 1996; 126: 2165 - 2174

10 Brian LT. Disease management of COPD with pulmonary rehabilitation. CHEST 1997; 112: 1630 - 1656

${ }^{11}$ British Thoracic Society Statement. COPD: summary of guidelines. Thorax 1997; 52 (Suppl 5): S2 - 21

${ }^{12}$ Brochard L, Mancebo J, Wysocki M, Lofaso F, Conti G et al.. Noninvasive ventilation for acute exacerbations of chronic obstructive pulmonary disease. NEJM 1995; 333: 817 - 822

${ }^{13}$ Büchi S, Villiger B, Sensky T, Schwarz F, Wolf Ch, Buddeberg C. Psychosocial predictors of long-term success of in-patient pulmonary rehabilitation of patients with COPD. Eur Respir J 1997; 10: 1272 - 1277

${ }^{14}$ Burge PS. EUROSCOP, ISOLDE and the Copenhagen city lung study. Thorax 1999; 54: 287 - 288

${ }^{15}$ Chambers DC, Tunnicliffe WS, Ayres JG. Acute inhalation of cigarette smoke increases lower respiratory tract nitric oxide concentrations. Thorax 1998; 53: 677 - 679

${ }^{16}$ Chanez P, Vignola AM, O'Shaugnessy R, Enander I, Li D, Jeffery PK, Bousquet J. Corticosteroid reversibility in COPD is related to features of asthma. Respir Crit Care Med 1997; 155: 1529 - 1534

17 COMBIVENT Inhalation Aerosol Study Group. In chronic obstructive pulmonary disease, a combination of ipratropium and albuterol is more effective than either agent alone. CHEST 1994; 105: 1411 - 1419

${ }^{18}$ Confalonieri M, Mainardi E, Porta RD, Bernorio S, Bandola L, Beghè $\mathrm{B}$, Spanevello $\mathrm{A}$. Inhaled corticosteroids reduce neutrophilic bronchial inflammation in patients with chronic obstructive pulmonary disease. Thorax 1998; 53: 583 - 585

19 Doll R, Peto R, Wheatley K, Gray R, Sutherland I. Mortality in relation to smoking: 40 years observation on male British doctors. BMJ 1994; 309: 901 - 910

${ }^{20}$ Gosselink R, Troosters T, Decramer M. Exercise training in COPD patient: the basic questions. Eur Respir J 1997; 10: 2884 - 2891

${ }^{21}$ Hak E, Essen van GA, Buskens E, Stalman W, de Melker RA. Is immunising all patients with chronic lung disease in the community against influenza cost effective? J Epidem and Community Health 1998; 52: $120-125$
${ }^{22}$ Henningsfield JA. Nicotine medications for smoking cessation. N Engl J Med1196 - 1203

${ }^{23}$ Hess Th. Pneumokokken-Impfung - Ja oder Nein? Schweiz Med Wochenschr 1096 - 1103

${ }^{24}$ Hillberg RE, Johnson DC. Noninvasive Ventilation. N Engl J Med 1997; 337: 1746 - 1752

${ }^{25}$ Hurt RD, Sachs DPL, Glover ED. A comparison of sustainedrelease bupropion and placebo for smoking cessation. $\mathrm{N}$ Engl J Med 1997; 337

${ }^{26}$ Ingenito EP, Evans RB, Loring SJ, Kaczka DW, Rodenhouse JD, Body SC, Sugarbaker DJ, Mentzer SJ, DeCamp MM, Reilly JJ. Relation between preoperative inspiratory lung resistance and the outcome of lung-volume-reduction surgery for emphysema. N Engl J Med 1998; 338: 1181 - 1185

${ }^{27}$ Jeffery PK. Structural and inflammatory changes in COPD: a comparison with asthma. Thorax 1998; 53: 129 - 136

${ }^{28}$ Joint ACCP/ACVPR Evidence-Based Guidelines:. Pulmonary Rehabilitation. CHEST 1997; 112: 1363 - 1396

${ }^{29}$ Jones PW, Bosh TK in association with an international study group. Quality of life changes in COPD patients treated with Salmeterol. Am J Respir Crit Care Med 1997; 155: 1283 - 1289

${ }^{30}$ Keatings VM, Jatakanow A, Worsdell YM, Barnes PJ. Effects of inhaled and oral glucocorticoids on inflammatory indices in asthma and COPD. Am J Respir Crit Care Med 1997; 155: 542 548

${ }^{31}$ Köhler D, Criée C, Raschke F et al. Leitlinien zur häuslichen Sauerstoff- und Heimbeatmungstherapie. Med Klinik 1997; 92: $2-6$

${ }^{32}$ Koyama H, Geddes DM. Genes, oxidative stress, and the risk of chronic obstructive pulmonary disease. Thorax 1998; 53 (Suppl 2): S10 - S14

${ }^{33}$ Langley SJ, Masterson CM, Batty EP, Woodcock A. Bronchodilator response to salbutamol after chronic dosing with salmeterol or placebo. Eur Respir J 1998; 11: 1081 - 1085

34 Leuenberger P, Anderhub HP, Brändli O, Keller R, Knoblauch A, Kuhn M, Perruchoud AP, Rochat T, Russi E, Villinger B, Zellweger JP. Management 1997 of chronic obstructive pulmonary disease. Schweiz Med Wochenschr 1997; 127: 766 - 782

${ }^{35}$ Leuenberger P, Schwartz J, Ackermann-Liebrich U, Blaser K, Bolognini $G$ et al. Passive Smoking Exposure in Adults and Chronic Respiratory Symptoms (SAPALDIA Study). Am J Resp Crit Care Med 1994; 150: 1221 - 1228

${ }^{36}$ Leuppi JD, Zenhäusern R, Schwarz F, Frey WO, Villiger B. Bedeutung der Trainingsintensität für die Verbesserung der Ausdauerleistungsfähigkeit bei Patienten mit chronisch obstruktiven Lungenerkrankungen. Dtsch Med Wschr 1998; 123: 174 178

${ }^{37}$ Llewellyn-Jones CG, Harris TAJ, Stockley RA. Effect of fluticasone propionate on sputum of patients with chronic bronchitis and emphysema. Am J Respir Crit Care Med 1996; 153: 616 - 621

${ }^{38}$ Lundback B, Lindstrom M, Jonsson E, Anderson S, van Herwaarden $\mathrm{C}$. Effect of $\mathrm{N}$-acetylcysteine on the decline in lung function in patients with COPD. Eur Respir J 1995; 5 (Suppl 15): 895

${ }^{39}$ Maesen FPV, Smeets JJ, Siedsens TJH, Wald FDM, Cornelissen PJG. Tiotropium bromide, a new long-acting antimuscarinic bronchodilator: a pharmacodynamic study in patients with chronic obstructive pulmonary disease (COPD). Eur Respir J 1995; 8: 1506 - 1513

${ }^{40}$ Maziak W, Loukides S, Culpitt S, Sullivan P, Kharitonov SA, Barnes PJ. Exhaled nitric oxide in chronic obstructive pulmonary disease. Am J Respir Crit Care Med 1998; 157: 998 - 1002

${ }^{41}$ McEvoy CE, Ensrud KE, Genant HK, Uy W, Griffith JM, Niewoehner DE. Association between corticosteroid use and vertebral fractures in older men with chronic obstructive pulmonary disease. Am J Respir Crit Care Med 1998; 157: 704 - 709 
42 McEvoy CE, Niewoehner DE. Adverse effects of corticosteroid therapy for COPD. A critical review. CHEST 1997; 111: 732 - 743

${ }^{43}$ Paggiaro PL, Dahle R, Bakran I, Frith L, Hollingworth K et al. Multicentre randomised placebo-controlled trial of inhaled fluticasone propionate in patients with chronic obstructive pulmonary disease. Lancet 1998; 315: $773-780$

44 Pauwels RA, Löfdahl C-G, Laitinen LA, Schouten JP, Postma DS et al. Longterm treatment with inhaled budesonide in persons with mild chronic obstructive pulmonary disease who continue smoking. NEJM 1999; 349: 1948 - 1953

${ }^{45}$ Perrin C, El Far Y, Vandenbos F, Tamisier R, Dumon MC, Lemoigne F, Mouroux J, Blaive B. Domiciliary nasal intermittent positive pressure ventilation in severe COPD: effects on lung function and quality of life. Eur Respir J 1997; 10: 2835 - 2839

${ }^{46}$ Petty TL. Supportive Therapy in COPD. CHEST 1998; 113: 256S 262S

${ }^{47}$ Rabe KF, Dent G, Magnussen H. Theophyllin und selektive Phosphodiesterase-Hemmer in der Therapie obstruktiver Atemwegserkrankungen. Pneumologie 1997; 51: 303 - 316

${ }^{48}$ Renkama TEJ, Schouten JP, Koëter GH, Postma DS. Effects of long-term treatment with corticosteroids in COPD. CHEST 1996; 109: 1156 - 1162

${ }^{49}$ Rennard SI. Overview of definitions, epidemiology, and factors influencing its development. CHEST 1998; 113: 235 - 241S

${ }^{50}$ Rennard SI, Serby CW, Ghafouri M, Johnson PA, Friedman M. Extended therapy with ipratropium is associated with improved lung function in patients with COPD. CHEST 1996; 110: 62 - 70

${ }^{51}$ Repine JE, Bast A, Lankhorst I and The Oxidative Stress Study Group. Oxidative stress in chronic obstructive pulmonary disease. Am J Respir Crit Care Med 1997; 156: 341 - 357

${ }^{52}$ Russi EW, Stammberger U, Weder W. Lung volume reduction surgery for emphysema. Eur Respir J 1997; 10: 208 - 218

${ }^{53}$ Sandford AJ, Weir TD, Paré PD. Genetic risk factors for chronic obstructive pulmonary disease. Eur Respir J 1997; 10: 1380 1391

54 Sciurba FC, Rogers RM, Keenan RJ, Slivka WA, Gorcsan J, Ferson PF, Holbert M, Brown ML, Landreneau RJ. Improvement in pulmonary function and elastic recoil after lung-reduction surgery for diffuse emphysema. N Engl J Med 1996; 334: 1095 1099

${ }^{55}$ Senior MR, Anthonisen MR. Chronic obstructive pulmonary disease (COPD). Am J Respir Crit Care Med 1998; 157: S139 S147

56 Siafakas NM, Vermeire P, Pride NB, Paoletti P, Gibson J et al. Optimal assessment and management of chronic obstructive pulmonary disease (COPD). ERS-consensus statement. Eur Respir J 1995; 8: 1398 - 1420

57 Silverman EK, Chapman HR, Drazen JM, Weiss ST, Rosner B et al. Genetic epidemiology of severe, early-onset chronic obstructive pulmonary disease. Am J Respir Crit Care Med 1998; 157: 1770 1778

58 Sivasothy P, Smith IE, Shneerson JM. Mask intermittent positive pressure ventilation in chronic hypercapnic respiratory failure due to chronic obstructive pulmonary disease. Eur J Respir 1998; 11: $34-40$

${ }^{59}$ Smith CA, Harrison DJ. Association between polymorphism in gene for microsomal epoxide hydrolase and susceptibility to emphysema. Lancet 1997; 350: 630 - 633

${ }^{60}$ Speich R, Boehler A, Weder W. Lungentransplantation bei fortgeschrittenem Lungenemphysem. Therapeutische Rundschau 1999; 56(3): $161-165$

61 Stammberger U, Bloch KE, Thurnheer R, Bingisser R, Weder W, Russi EW. Exercise performance and gas exchange after bilateral video-assisted thoracoscopic lung volume reduction for severe emphysema. Eur Respir J 1998; 12: 785 - 792
62 Stanescu D, Sanna A, Veriter C, Robert A. Identification of smokers susceptible to development of chronic airflow limitation. A 13-year follow up. CHEST 1998; 114: 416 - 425

${ }^{63}$ Thompson WH, Nielson CP, Carvalho P, Charan NB, Crowley JJ. Controlled trial of oral prednisone in outpatients with acute COPD exacerbation. Am J Respir Crit Care Med 1996; 154: 407 412

${ }^{64}$ Ukena D, Harnest U, Sakalauskas R, Magyar P, Vetter N et al. Comparison of addition of theophylline to inhaled steroid with doubling the dose of inhaled steroid in asthma. Eur Respir J 1997; 10: 2754 - 2760

65 Ullmer E, Bolliger CT. Tabak: Asche, Rauch und „Kohle“. Schweiz Med Wochenschr 1999; 129: $102-112$

${ }^{66}$ Ullmer E, Solèr M. Asthma und COPD: Unterschiede und Gemeinsamkeiten. Internist 1999; 40: 837 - 843

${ }^{67}$ Vestbo J, Sorensen T, Lange P, Brix A, Torre P, Viskum K. Longterm effect of inhaled budesonide in mild and moderate chronic obstructive pulmonary disease: a randomised controlled trial. Lancet 1999; 353: 1819 - 1823

${ }^{68}$ Wettengel R, Böhning W, Cegla U, Criée C, Fichter J et al. Empfehlungen der Deutschen Atemwegsliga zur Behandlung von Patienten mit chronisch obstructiver Bronchitis und Lungenemphysem. Med Klinik 1995; 90: 3 - 7

${ }^{69}$ Wilson R. The role of infection in COPD. CHEST 1998; 113: $242 S-248 S$

${ }^{70}$ Zemp E, Elsasser S, Schindler Ch, Künzli N, Perruchoud AP et al. Long-Term Ambient Air Pollution and Respiratory Symptoms in Adults (SAPALDIA Study). Am J Respir Crit Care Med 1999; 159: $1257-1266$

71 Zielinski J, Tobiasz M, Hawrylkiewicz I, Sliwinski P, Palasiewicz G. Effects of long-term oxygen therapy on pulmonary hemodynamics in COPD patients. A 6-year prospective study. CHEST 1998; $113: 65-70$

Prof. Dr. med. André P. Perruchoud

Chefarzt Innere Medizin DIM A

Kantonsspital Basel

Petersgraben 4

4031 Basel

Schweiz 